\title{
Aportes a la sustentabilidad de la cadena de valor de frutos secos: obtención de aceite y harina de nuez (Juglans regia L.) y almendra (Prunus amygdalus L.)
}

\section{Marcela Lilian Martínez ${ }^{a, b, c}$, María Cecilia Penci ${ }^{a, c}$, Damián Modesto Maestri ${ }^{a, b}$, Pablo Daniel Ribotta ${ }^{c, d}$ y María Andrea Marín ${ }^{a, c^{*}}$}

\footnotetext{
${ }^{a}$ Instituto de Ciencia y Tecnología de los Alimentos (ICTA), Facultad de Ciencias Exactas, Físicas y Naturales (FCEFyN), Universidad Nacional de Córdoba (UNC), Argentina

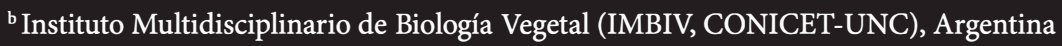

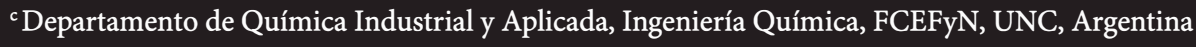

d Instituto de Ciencia y Tecnología de Alimentos Córdoba (CONICET-UNC), Argentina

*Email: ma.andrea.marin@gmail.com
}

\section{RESUMEN}

Recepción: 2012-03-21

Aceptación: 2012-05-24

Los frutos secos, nuez (Juglans regia L.) y almendra (Prunus amygdalus L.), constituyen alimentos energéticos y nutritivos con un contenido elevado de ácidos grasos esenciales y pueden ser utilizados como alimento y para la formulación de cosméticos. En el marco de la sustentabilidad de la cadena de valor y considerando las premisas del desarrollo sostenible, se extrajo el aceite mediante prensa de tornillo helicoidal (a escala piloto e industrial). Esta metodología resulta adecuada para obtener altos rendimientos de aceite y de buena calidad química sin utilizar solventes. No obstante, por su elevado nivel de insaturación, los aceites son altamente susceptibles al deterioro oxidativo. La adición de antioxidantes, naturales y artificiales, y el empleo de procedimientos físicos de protección constituyen alternativas tecnológicas para su conservación.

Las experiencias de extracción por prensado a escala piloto permitieron determinar, para ambos frutos, el rango de tamaño de partícula $(2.4-4.8 \mathrm{~mm})$ de la alimentación de la prensa y el contenido de agua inicial para lograr la máxima recuperación de aceite. Se obtuvieron rendimientos cercanos al $90 \%$ en peso $\left(7.5 \%\right.$ de humedad y $50{ }^{\circ} \mathrm{C}$ ) y al $80 \%$ en peso ( $8 \%$ de humedad y $40{ }^{\circ} \mathrm{C}$ ) para nuez y almendra, respectivamente. Estas condiciones permitieron obtener residuos de extracción de nuez y almendra con 25 y $21 \%$ en peso de proteínas totales, respectivamente. Estos sólidos fueron utilizados en el desarrollo de fideos laminados frescos. Estos productos presentaron excelentes propiedades tecnológicas tanto los elaborados con un reemplazo 
de hasta el $30 \%$ en peso de la harina de trigo con harina de frutos secos, como en el caso de los fideos libres de gluten. En este último caso, la mejor formulación fue la compuesta por $40 \%$ en peso de harina de frutos secos, $45 \%$ en peso de harina de soja, almidón de maíz y gomas.

Entre los antioxidantes ensayados para evaluar la estabilidad oxidativa de los aceites, el TBHQ (butilhidroquinona terciaria), solo o combinado, presentó la mayor eficacia antioxidante, aún con la mitad de la concentración máxima permitida por el Código Alimentario Argentino. Los antioxidantes naturales analizados (extracto de romero y palmitato de ascorbilo), si bien no lograron la eficacia del TBHQ, ofrecen una alternativa que debe ser más explorada, teniendo en cuenta efectos sinérgicos que pueden contribuir a estabilizar este tipo de aceites utilizando menos antioxidantes sintéticos.

Palabras clave: nuez, almendra, prensado en frío, estabilidad del aceite, residuo de extracción, harina.

\section{ABSTRACT}

The dried fruits, nuts (Juglans regia L.) and almond (prunus amygdalus L.), are energetic and nutritious foods with a high content of essential fatty acids that can be used as foods and for the elaboration of cosmetics. As part of the sustainability and the value chain, and taking into consideration the premises of sustainable development, the oil was extracted using the helical screw press (to pilot and industrial scale). This method is suitable to obtain high yields of quality oil and chemicals without using solvents. However, due to its high levels of unsaturation, the oils are highly perceptive to oxidative deterioration. Adding natural and artificial antioxidants and using protective physical procedures for its protection are technological alternatives for its conservation. The extraction by pilot scale press allowed determining, for both fruits, the particle size range (2.4-4.8 mm.) of the press feeding and the initial content of water for a maximum oil recovery. There was a yield of nearly $90 \%$ in weight $\left(7.5 \%\right.$ humidity and $\left.50{ }^{\circ} \mathrm{C}\right)$ and $80 \%$ in weight $\left(8 \%\right.$ humidity and $40{ }^{\circ} \mathrm{C}$ ) for nuts and almond, respectively. These conditions allowed to obtain extraction residues for nuts and almonds with 25 and $21 \%$ of weight of total proteins respectively. These solids were used in the production of rolled fresh noodles. These products presented excellent technological properties, like the ones made with a substitution of up to $30 \%$ in weight of wheat flour of dried fruits, like the gluten-free noodles. In this last case, the 
best formula was the one composed by $40 \%$ in weight of dried fruit flour, $45 \%$ in weight of soy flour, corn starch and gum.

Among the antioxidants tested to evaluate the oxidative stability of the oils, the TBHQ (butilhidroquinona tertiary), alone or combined, presented the highest antioxidant efficacy, even with half of the maximum allowed concentration by the Argentinian Food Code. The natural antioxidants analyzed (rosemary extract and ascorbyl palmitate), even though did not reached the maximum efficacy of TBHQ, offer an alternative that must be more exploited, taking into account the synergic effects that can contribute to stabilize this type of oils using less synthetic antioxidants.

Key words: nuts, almond, cold-pressed, oil stability, extraction residues, flour.

\section{INTRODUCCIÓN}

Dentro del ámbito internacional, la Argentina ocupa un lugar poco destacado en la producción general de frutos secos. Sin embargo, comienza a participar más con volúmenes crecientes de producción de nueces e incrementos de superficies plantadas de almendro, pecan y avellano. La producción mundial de nuez (Juglans regia L.), durante el periodo 2006/07 - 2010/11, ha tenido un incremento de 45\%. Desde el año 2006/07 la producción mundial creció constantemente, estimándose para la temporada 2010/11 un total de $1.405 .060 \mathrm{t}$.

El nogal es la principal especie entre los frutos secos cultivados en Argentina, con una superficie plantada de 14.000 ha y una producción cercana a las 11.000 t. Esta producción se ha registrado como resultado de la entrada en producción de nuevas plantaciones, de aquellas reconvertidas y de la entrada en producción de las tradicionales beneficiadas por condiciones ambientales favorables. El $63 \%$ de esa producción se concentra en las provincias de Catamarca (38\%) y Mendoza (24\%). El resto se encuentra distribuido entre La Rioja (21\%), San Juan (11\%), Río Negro (2\%) y otras provincias (3\%). Cada zona productora posee diferentes características en el manejo del cultivo, las variedades y las formas de comercialización.

En los últimos 20 años, la superficie de cultivo de almendra (Prunus amygdalus $\mathrm{L}$ ), por su 
parte, creció en forma sostenida, pasando de 1.200.000 a 1.600 .000 ha, llegando en el año 2003 a 1.700 .000 ha. La producción nacional de almendras ronda las $1.200 \mathrm{t}$ y resulta insuficiente para abastecer la demanda interna. La almendra se cultiva principalmente en las provincias de Mendoza (1.430 ha) y San Juan (550 ha). Con la aplicación de la ley de diferimientos impositivos, la superficie plantada se incrementaría entre 1.000 y 1.200 ha en los próximos años, para alcanzar una producción de $3.200 \mathrm{t}$ de pepita de almendra (Iannamico, 2011; MinAgri, 2011).

No obstante, el crecimiento de estos sectores productivos no ha sido acompañado en forma adecuada por un conocimiento científico riguroso de importantes aspectos, que hacen a la calidad de las materias primas y, en consecuencia, de los subproductos elaborados. El interés desde el punto de vista científico y tecnológico es enorme, puesto que a la vez que estos conocimientos propenden a la sustentación del cultivo, se buscan nuevos desarrollos con la finalidad de otorgarle mayor valor agregado a la producción, en un marco de aprovechamiento y desarrollo sostenible del sector.

El valor comercial de los frutos secos o "nuts" radica fundamentalmente en la utilización de la pulpa o semilla que se consume fresca, tostada o salada, en confituras, pastelería, panadería u otros usos culinarios.
Estos frutos no sólo son ricos en energía ya que contienen entre 50 y $70 \%$ de aceite, el cual se encuentra altamente insaturado, sino que también son fuente de proteínas, hidratos de carbono, fibras, minerales, vitaminas y otros compuestos hidrosolubles como polifenoles y pigmentos.

La nuez (Juglans regia L.) se destaca por poseer un elevado contenido de aceite (60 $74 \%)$, con uno de los más altos porcentajes conocidos de ácidos grasos poliinsaturados (linoleico, 52 - 60\% y linolénico, 12 - 20\%) (Prasad, 2003; Crews et al., 2005; Martínez et al., 2006, 2008 a; Martínez \& Maestri, 2008). La semilla contiene entre 13 y $18 \%$ de proteínas, 12 - 16\% de hidratos de carbono, 1.5 - $2.0 \%$ de fibras, 1.7 - 2.0\% de minerales, vitaminas y otros compuestos hidrosolubles como ácido fítico, polifenoles y pigmentos (Wardlaw, 1999; Lavedrine et al., 2000; SzeTao \& Sathe, 2000; Li et al., 2006; Labuckas et al., 2008; Zhang et al., 2009). La almendra (Prunus amygdalus L.) contiene de 48 a 67\% de aceite, con un elevado contenido de ácido oleico (63-78\%) y linoleico (12 - 27\%) (Cherif et al., 2004; Kodad \& Socias, 2008; Moayedi et al., 2010). La semilla contiene además entre $12-20 \%$ de proteínas, $20 \%$ de hidratos de carbono, minerales y vitaminas (Ahmad, 2010; Ozcan et al., 2011).

La producción de aceites comestibles ha mostrado en los últimos años un crecimiento 
sostenido nacional e internacional y, en la actualidad, es una actividad económica de gran relevancia. Dentro de este gran rubro de la economía, la elaboración de aceites no tradicionales, como los de nuez y almendra, ofrecen una oportunidad que no ha sido prácticamente explotada. Entre los subproductos de la industria aceitera, la harina o residuo de extracción se presentan como una alternativa novedosa para un mercado mundial que requiere productos saludables de alto valor nutricional.

El bajo volumen de producción hace difícil que estos aceites, así como sus residuos de extracción, puedan transformarse en productos de uso masivo. Pero poseen un gran potencial para producir alimentos para necesidades específicas, debido a los beneficios que trae aparejado su consumo en el tratamiento de algunas enfermedades no transmisibles, principalmente enfermedades cardiovasculares, diabetes y cáncer (Laverdrine et al., 1999; Chen et al., 2006).

Uno de los principales problemas para la producción de estos aceites no tradicionales radica en encontrar el método de extracción adecuado que provea buenos rendimientos de extracción y calidad de aceite, para determinar la viabilidad de su producción comercial.
De entre las tecnologías de extracción, en los últimos años, se ha intensificado el interés por la obtención de aceites a través de tecnologías de prensado con prensa hidráulica y de tornillo. Este tipo de tecnología provee un método sencillo para obtener aceites de pequeños lotes de semillas, (Wiesenborn et al., 2001; Moure et al., 2002; Singh et al., 2002; Zheng et al., 2003; Zuñiga et al., 2003; Soto et al., 2007; Martinez et al., 2008; Uquiche et al., 2008). Esto resulta apropiado para materiales con alto contenido en aceite, además requiere instalaciones menos costosas e involucra operaciones más seguras y menos riesgosas para el ambiente que la extracción por solvente. A su vez, los residuos de extracción que se generan como subproductos del proceso de obtención del aceite por prensado, presentan ácidos grasos poliinsaturados esenciales, linoleico y linolénico, alto contenido de proteínas, fibra alimentaria, minerales, vitaminas y sustancias con capacidad antioxidante como polifenoles y pigmentos. Su composición los convierte en una fuente potencial de nutrientes para ser la producción de alimentos con actividad beneficiosa sobre la salud.

Las pastas alimenticias son productos que se consumen en todo el mundo y se caracterizan por ser un alimento tradicional de gran aceptación (Petitot et al., 2009). Dentro de las muchas razones que justifican la popularidad de éstas se destacan, entre las más importantes, 
su ajustado perfil nutricional y el hecho de ser una fuente importante de carbohidratos complejos, moderada en proteínas y portadora de algunas vitaminas. La calidad de las pastas está relacionada directamente con sus propiedades de cocción. Estas son el resultado de las características de la materia prima y de las condiciones de elaboración. La calidad de las pastas cocidas se expresa en términos de absorción de agua, de material lixiviado durante la cocción y de los atributos relacionados a la textura, la firmeza y la pegajosidad. La textura de la pasta cocida es generalmente reconocida como su aspecto de calidad más importante (Brunnel et al., 2010). Las pastas han ganado popularidad por sus propiedades nutricionales al reconocerse como un producto con bajo índice glucémico. La liberación de los productos de degradación del almidón es menor en pastas que en otros productos de cereales, lo que es atribuido a la estructura compacta de la pasta, resultante del proceso de extrusión que deja una red de proteínas muy cerrada, atrapando los gránulos de almidón y retardando el ataque de amilasas (Tudorica et al., 2002). Además, las pastas son un buen vehículo para la incorporación de sustancias beneficiosas para la salud, ya que son productos de fácil elaboración, bajo precio, larga vida útil y de rápida preparación (Dick \& Matsuo, 1988). La WHO (The World Health Organization) y la FDA (Food and Drug Administration) consideran a las pas- tas como un buen vehículo para la adición de nutrientes.

Los aceites con altos niveles de insaturación, como los de nuez y almendra, son susceptibles de sufrir procesos oxidativos que alteran su calidad química y organoléptica, a pesar del efecto protector de las sustancias antioxidantes presentes naturalmente en los mismos. Estas reacciones de deterioro de los lípidos constituyen una de las causas de mayor importancia comercial en la industria alimentaria, por las pérdidas que producen en grasas, aceites y alimentos que los contienen.

La adición de antioxidantes, en su mayor parte sintéticos, es un procedimiento tecnológico habitual en la industria alimentaria, ya que mejora la estabilidad de los lípidos y prolonga la vida útil de los alimentos que los contienen. No obstante, a la luz de las nuevas tendencias en alimentación, la presencia de estos aditivos sintéticos está siendo cuestionada cada vez en mayor grado. Muchas sustancias naturales, entre las que se encuentran fenoles simples, ácidos fenólicos, carotenoides, antocianinas y flavonoides, presentan propiedades antioxidantes (Moure et al., 2000; Gu et al., 2003; Goli et al., 2005; Yu et al., 2005; Alasalvar et al., 2006; Maestri et al., 2006; Kamath \& Rajini, 2007). Los mecanismos por los cuales estas sustancias ejercen su actividad son diferentes y su eficacia se ve influenciada notablemente

Volumen No. 1 Aportes a la sustentabilidad de la cadena de valor de frutos secos: obtención de aceite y harina de nuez (Juglans regia L.) y almendra (Prunus amygdalus L.) 
por las características del sustrato (Yanishlieva \& Marinova, 2001; Frankel, 2005). También, la mayor parte de las investigaciones han sido llevadas a cabo en sistemas modelo constituidos por lípidos puros, siendo escasas las contribuciones realizadas sobre matrices alimenticias complejas.

\section{MATERIALES Y MÉTODOS}

\subsection{Material vegetal}

Se utilizaron granos enteros de nuez y almendra de la variedad Franquette y Guara, respectivamente. De igual forma se incorporó la harina de trigo calidad panadera (sin aditivos).

\subsection{Extracción de los aceites}

Se utilizó una prensa de tornillo a escala piloto (Komet, Modelo CA 59 G, Figura 1). Se usó un diseño experimental factorial variando la temperatura de prensado y contenido de humedad del material. El tamaño de partícula estuvo comprendido en el rango de $2.4-4.8 \mathrm{~mm}$, el diámetro de la restricción del barral fue de $5 \mathrm{~mm}$ y la velocidad de prensado se mantuvo a $20 \mathrm{rpm}$ (Martínez et al., 2008). La humidificación del material se llevó a cabo mediante aspersión de agua según la metodología propuesta por Singh et al. (2000). Se determinó la calidad química de los aceites obtenidos en cada uno de los tratamientos realizados.

Figura 1- Extracción de aceite:

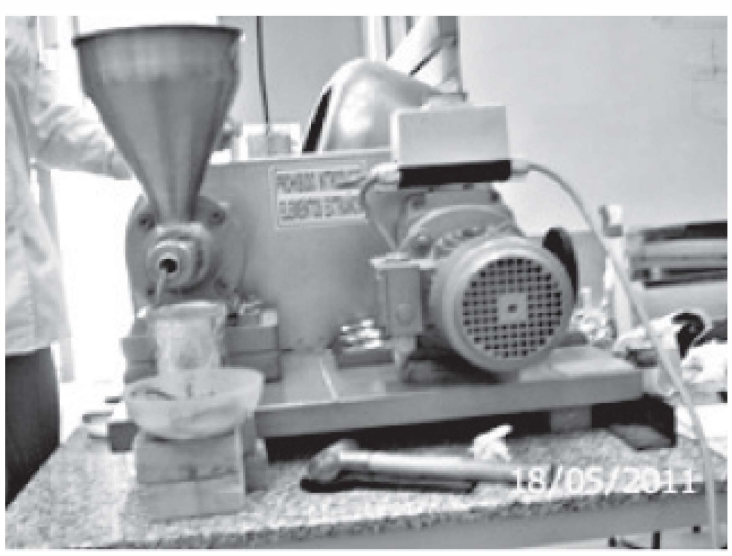

a) Prensa de tornillo

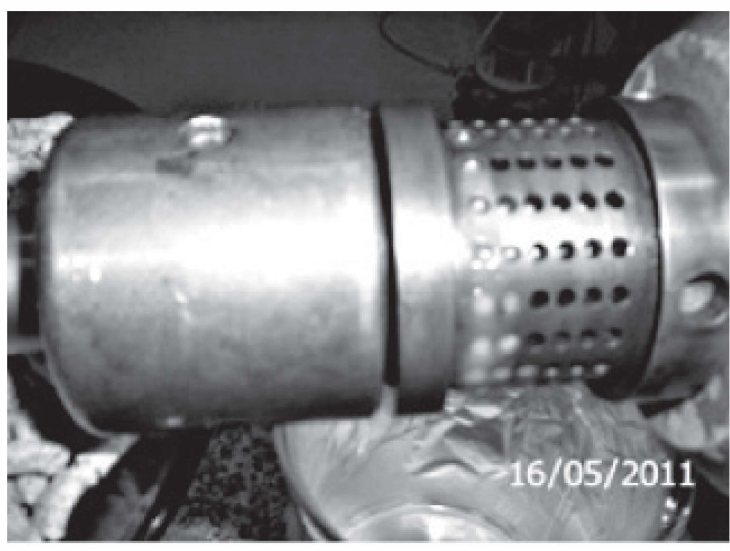

b) Residuo sólido,

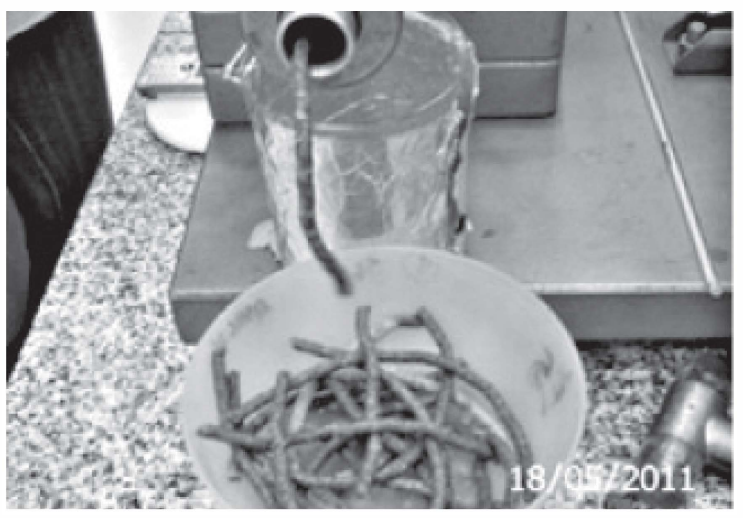

c) Extracto 


\subsection{Métodos analíticos para evaluar el proceso extractivo y la calidad química de los aceites obtenidos}

El rendimiento total en aceite, cantidad de aceite retenido en el residuo sólido $y$ el contenido de finos en el extracto se determinaron según metodología propuesta por Martínez et al. (2008).

Para la determinación de los índices generales decalidad delos aceitessesiguióla metodología descripta en AOCS (2009) en acidez, peróxidos y prueba espectrofotométrica en el UV. La cuantificación de los tocoferoles totales y evaluación de la capacidad antioxidante de los aceites se determinó siguiendo la metodología descrita por Wong et al. (1995) y Martínez et al. (2008), respectivamente.

\subsection{Evaluación de la eficiencia antio- xidante de sustancias naturales sobre la es tabilidad de los aceites}

Las sustancias antioxidantes seleccionadas, butilhidroquinona terciaria (TBHQ), palmitato de ascorbilo (PA), extracto de romero (ER) para realizar el estudio, se agregaron a los aceites, solas o en mezclas binarias o ternarias, de acuerdo a las concentraciones permitidas por el Código Alimentario Argentino.

\section{a. Obtención de las harinas deslipidiza- das}

El residuo de extracción se deslipidizó por extracción continua sólido-líquido en equipo Soxhlet, durante $12 \mathrm{~h}$, utilizando como disolvente n-hexano. El contenido de aceite se cuantificó por diferencia de pesos antes y después de la extracción (AOCS, 2009). Finalmente, el residuo de extracción deslipidizado se tamizó para conseguir el tamaño de partícula deseado para los ensayos posteriores (malla estándar No. 40 US).

\section{b. Preparación de las pastas}

Los ingredientes secos tamizados (harina de trigo, de nuez o almendra, harina de soja, almidón de maíz, gomas) se mezclaron con agua con sal e inmediatamente se amasó la pasta con una amasadora manual Philips HR 1495 a máxima velocidad, durante $3 \mathrm{~min}$. Se tomó la masa con las manos, conformando un bollo más o menos uniforme y se dejó reposar por $10 \mathrm{~min}$. El bollo fue laminado con una fabricadora de pastas Drago, $14 \mathrm{~cm}$ de longitud de ancho de lámina, con apertura regulable de los rodillos del 1 al 7. El bollo fue laminado-plegado-laminado con seis pasadas en el punto $\mathrm{n}^{\circ} 7 \mathrm{y}$ una pasada en los puntos $\mathrm{n}^{\circ} 6, \mathrm{n}^{\circ} 5 \mathrm{y} \mathrm{n}^{\circ} 4$. Seguidamente se cortaron los fideos de $2 \mathrm{~mm}$ de espesor e inmediatamente fueron colocados en bolsas de polietileno, las 
cuales se cerraron herméticamente por termo sellado. Las muestras de fideos se colocaron en refrigerador $\left(6-8^{\circ} \mathrm{C}\right)$ hasta su utilización para los ensayos de calidad.

\section{c. Determinación de las propiedades tecnológicas de las pastas}

El tiempo óptimo de cocción, residuo de cocción y absorción de agua se determinaron según la norma AACC (1995).

\section{d. Análisis estadístico}

Los resultados se evaluaron estadísticamente mediante análisis de la varianza (ANOVA) a dos factores (temperatura de prensado y contenido de humedad del material). En aquellos casos donde se observaron diferencias estadísticamente significativas se utilizó un test a posteriori de comparación múltiple (LSD), a un nivel de significación del $0.05 \%$. Todos los tratamientos se realizaron por triplicado.

\section{RESULTADOS Y DISCUSIÓN}

Las experiencias de extracción por prensado a escala piloto indicaron que el contenido de agua resultó un factor fundamental para lograr la máxima recuperación de aceite, lográndose rendimientos de casi el 90 y $80 \%$ con materiales, conteniendo 7.5 y $8.0 \%$ de humedad, extraídos a 50 y $40{ }^{\circ} \mathrm{C}$ para nuez y almendra respectivamente. Para ambas drupas la influencia de la temperatura fue de menor significación (Martínez et al., 2008; Hadad et al., 2012). Resultados similares fueron reportados por Singh \& Bargale (1990 y 2000). Estos autores, trabajando en un rango de contenidos de humedad comprendido entre el 5 y $12 \%$, observaron máximos de extracción de aceite en materiales (semillas de lino " $L i$ num usitatissimum" y colza "Brassica napus") con 7.0 - 7.5\% de humedad. Más aún, Li et al. (1999) demostraron que la temperatura y humidificación antes del prensado provoca una expansión y ruptura de las estructuras celulares e incrementa la plasticidad y permeabilidad del material, lo que facilitaría la salida del aceite y el consiguiente aumento del rendimiento del prensado. Sin embargo, en el caso de la nuez, con el contenido de humedad indicado anteriormente, un aumento de la temperatura de 50 a $70^{\circ} \mathrm{C}$, provocó una merma en el rendimiento como consecuencia de la aglutinación y compactación del material en el interior del dispositivo de prensado, lo que daría origen a una matriz menos porosa y a una menor fluidez del aceite (Martínez et al., 2008).

En el contenido de sólidos en el extracto, Vargas-López et al. (1999) y Singh et al. (2002) han observado que el mismo se incrementa

Marcela Lilian Martínez / María Cecilia Penci / Damián Modesto Maestri / Pablo Daniel Ribotta / María Andrea Marín | Volumen No. 1 
al disminuir el contenido de humedad del material (semillas de Crambe "Crambe abyssinica"). Los incrementos de sólidos fueron desde 1.1 a $5.4 \%$ y desde 2.9 a $4.4 \%$, cuando el porcentaje de humedad $(\% \mathrm{H})$ disminuyó de 9.2 a $3.6 \%$ y de 12.1 a $4.3 \%$ respectivamente. En este estudio, el contenido de sólidos en el extracto de nuez varió entre 5.7 y $16.8 \%$. El menor valor fue obtenido a $7.5 \% \mathrm{H} \mathrm{y} 70{ }^{\circ} \mathrm{C}$. Mientras que, en los extractos de almendra el menor contenido de sólidos varió entre 2.6 y $28.6 \%$. El menor valor fue obtenido a $8 \%$ y 40 ${ }^{\circ} \mathrm{C}$. Esto podría deberse a que la torta se torna más compacta provocando que menos partículas sólidas atraviesen los orificios del barral y sean coextraídos con el aceite (Martínez et al., 2008; Hadad et al., 2012).

En todos los tratamientos, los aceites de nuez y de almendra, presentaron bajos niveles de acidez ( $<0.46$ y $0.16 \%$ ácido oleico, respectivamente), demostrando que no hay deterioro hidrolítico. No se detectaron peróxidos en ninguno de los aceites. El coeficiente de extinción específica $\mathrm{k}_{232}$, que se relaciona con la estabilidad oxidativa de los aceites y que corresponde a la máxima absorción de dienos conjugados, presentó valores inferiores a 1.17 y 1.08 , respectivamente, siendo estos valores habituales encontrados en aceites vegetales. El contenido de tocoferoles total varió entre 312 y $360 \mu \mathrm{g} / \mathrm{g}$ aceite de nuez y 561 y 610 $\mu \mathrm{g} / \mathrm{g}$ aceite de almendra. Las condiciones empleadas en los tratamientos no afectan prácticamente la composición de ácidos grasos ni la estabilidad oxidativa de los aceites. La distribución de ácidos grasos en el aceite de nuez fue: ácido linolénico (18:3), 15.2\%; ácido linoleico (18:2), 52.4\%; ácido oleico (18:1), $22.9 \%$; ácido esteárico (18:0), 2.14\%; ácido palmítico (16:0), 7.20\% y ácido palmitoleico (16:1), 0.08\%. Mientras que para el aceite de almendra fue: ácido linoleico (18:2), 19.8\%; ácido oleico (18:1), 71.2\%; ácido esteárico (18:0), 1.8\%; ácido palmítico (16:0), 6.7\% y ácido palmitoleico (16:1), $0.4 \%$ (Martínez et al., 2008; Hadad et al., 2012).

Dado que los aceites de nuez y almendra constituyen un sustrato altamente susceptible a reacciones oxidativas que deterioran su calidad química y organoléptica. Este hecho hace necesario que para su conservación deba ser protegido con sustancias o condiciones de almacenamiento que puedan inhibir o retardar los procesos de oxidación mencionados. El efecto protector del TBHQ sobre la estabilidad oxidativa de los aceites puede lograrse con la mitad de la concentración máxima permitida (200ppm) por el Código Alimentario Argentino para antioxidantes sintéticos en aceites vegetales. La valoración de la capacidad antirradicalaria de los aceites aditivados permitió determinar que: a) el TBHQ es un efectivo inhibidor de 
radicales libres; b) su actividad es dependiente de la concentración y no del tiempo; c) el palmitato de ascorbilo y el extracto de romero no ejercen un efecto aditivo sobre la actividad del TBHQ (Martínez et al., 2012; Menapace et al., 2012).

Las pastas obtenidas, a partir de las formulaciones diseñadas con harina de trigo y las libres de gluten, cumplen con las propiedades reológicas, de textura y calidad características para este tipo de productos.

En las formulaciones elaboradas con harina de trigo y con hasta $30 \%$ en peso de harina de nuez (parcial o totalmente deslipidizada), los tiempos óptimos de cocción variaron entre 5 y $6 \mathrm{~min}$, los cuales fueron similares a los tiempos normales para pastas frescas. La pérdida de materia durante la cocción varió entre 5.97 y $8.38 \%$ y la absorción de agua varió entre $120.8 \%$ y $161.9 \%$ y aumentó con la cantidad de sólidos de nuez. La absorción de agua durante la cocción fue afectada, al igual que el residuo generado, fundamentalmente por el porcentaje de sólidos de nuez en la formulación $(\mathrm{p} \leq 0.05)$. Las propiedades tecnológicas de las pastas obtenidas con los máximos niveles de sustitución de la harina de trigo se encontraron dentro de los parámetros informados habitualmente en este tipo de productos, lo que no generaría cambios significativos en el proceso industrial de producción.
Las pastas elaboradas a base de harina de almendra, soja y almidón de maíz presentaron tiempos óptimos de cocción entre 2 y 4 min. Este parámetro aumentó conforme al incremento de la proporción de la harina de almendra respecto a la de soja en las distintas formulaciones. La pérdida de masa durante la cocción obedeció fundamentalmente al contenido de almidón de maíz y harina de soja en la formulación ( $\mathrm{p} \leq 0.05)$. El residuo de cocción aumentó significativamente al disminuir la proporción de almidón de maíz y aumentar la de harina de soja, mientras la cantidad de harina de almendra permanecía constante. La absorción de agua resultó en todas las formulaciones con menor porcentaje de harina de almendra, inferior a las pastas comerciales libre de gluten y de trigo $(\mathrm{p} \leq 0.05)$.

Un resumen de las operaciones de la cadena de valor que se propone en el presente trabajo para la producción de frutos secos, aceite y harina se muestran en la figura 2 .

Asimismo, la figura 2 muestra la posibilidad de utilización de otros residuos como subproductos o materias primas de otras industrias como es el caso del mesocarpio, que puede ser transformado en colorante y el endocarpio que puede ser utilizado como insumo de la industria de los colorantes o como fuente para la obtención de carbón activado; generando una visión más integral de la cadena 
de valor posibilitando la sustentabilidad de la misma y el desarrollo sostenible.

En la actualidad, las cadenas de valor de la nuez y la almendra están centradas en la obtención de los frutos secos como producto.
El desarrollo de productos alternativos novedosos persigue la creación de valor agregado resaltando los beneficios nutricionales que estos frutos presentan. El análisis desde la visión sistémica de cadena de valor es una poderosa herramienta para la planificación es-

Figura 2: Diagrama de flujo desde la poscosecha de la nuez y almendra.

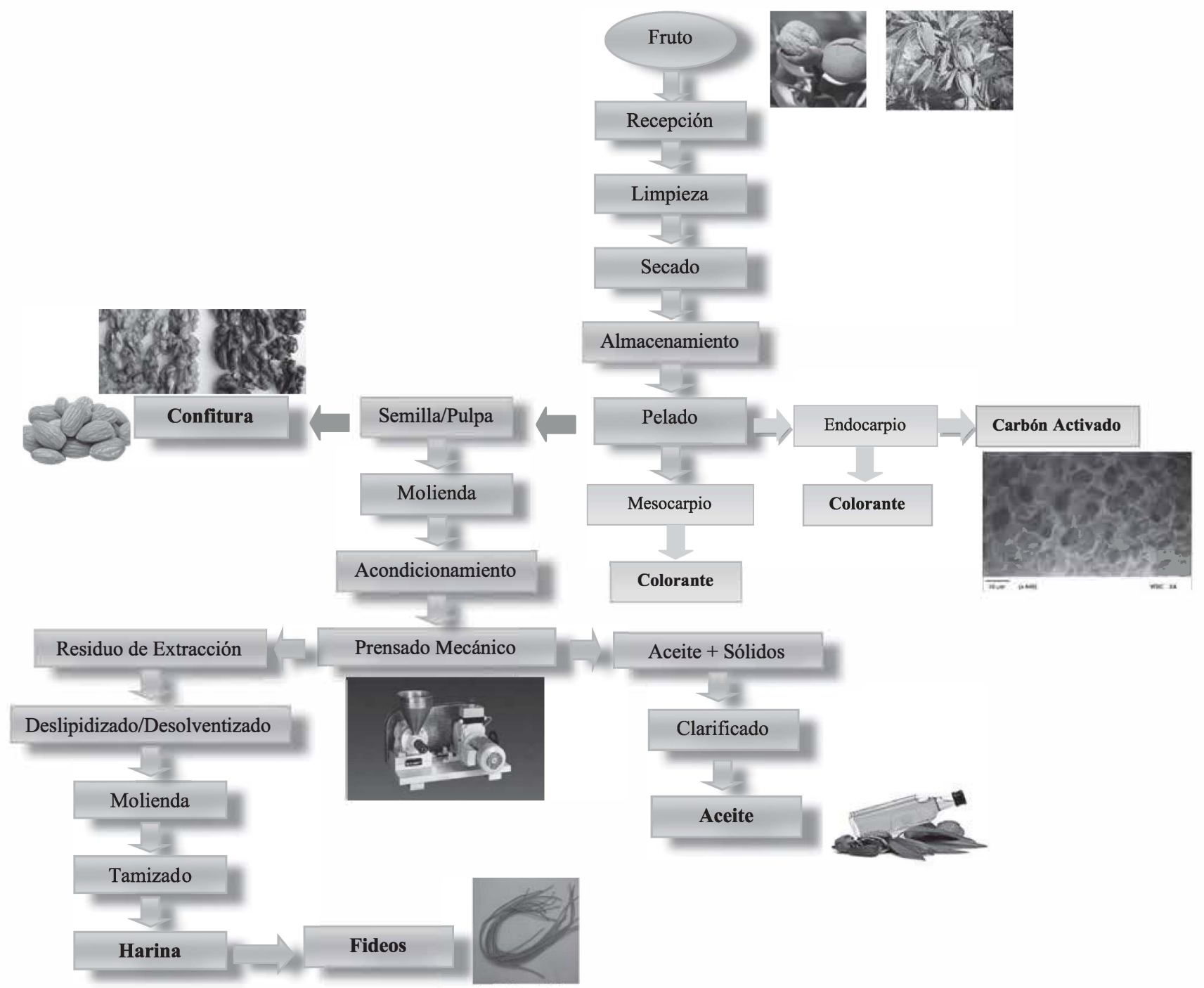

Volumen No. 1 Aportes a la sustentabilidad de la cadena de valor de frutos secos: obtención de aceite y harina de nuez (Juglans regia L.) y almendra (Prunus amygdalus L.) 
tratégica que permite maximizar la creación de valor, mientras se minimizan los costos sin perjuicio de la calidad química, nutricional y de inocuidad de los productos obtenidos. Por lo anteriormente dicho es que se consideró particularmente el límite tecnológico, pues en ocasiones ha afectado también la calidad de la oferta y el valor que ésta genera, considerando como prioritario satisfacer las necesidades actuales sin comprometer los recursos y posibilidades de las futuras generaciones.

Como ya se mencionó, las cadenas de valor de la nuez y la almendra se encuentran actualmente en una etapa de crecimiento desde una economía basada en la inversión para la generación de innovación, que permita posicionar la Cadena en su tránsito hacia una economía basada en el uso de métodos innovadores y eficientes. No obstante, es posible distinguir algunos agentes en la cadena que aún persisten en una economía basada en los factores, situación que no es sustentable en el tiempo, por lo cual se hace necesario avanzar en los distintos retos estratégicos, tales como: desvincular la ventaja competitiva de la cadena de la dependencia de los recursos naturales, a través de la inversión en el desarrollo e implementación de tecnologías innovadoras eficientes que permitan aumentar los rendimientos actuales en volumen y calidad; integrar el sector productivo con el sector de investigación y desarrollo para generar nue- vo conocimiento de innovación nacional e internacional y fomentar las relaciones entre agentes, asociaciones de intereses comunes, comercialización, marketing genérico, gestión administrativa y comercial, entre otras; de manera de aprovechar el potencial de los distintos agentes que poseen actitudes positivas hacia el desarrollo sostenible.

Para lograr éxitos productivos, además del material vegetal, debe contarse con un adecuado diseño de las plantaciones. El conocer bien la especie, su fenología y las perturbaciones que los cambios climáticos previsibles en una zona pueden provocar, ayuda a no cometer errores o a paliar los efectos de los factores limitantes que suelen existir en casi todas las áreas de cultivo. Por lo tanto, el proceso de producción primaria de los frutos secos consiste en la elección del lugar adecuado para que se generen las condiciones necesarias para que el cultivo exprese su potencial. La elección varietal no sólo está condicionada por el factor climático sino también por el productivo y el mercado. El diseño de la plantación se encara siempre a minimizar el efecto de los factores limitantes de la zona; aunque como aspectos básicos se consideran: la elección de un buen material de partida que garantice la homogeneidad en crecimiento y facilite la formación, la adecuada combinación varietal, la disposición de los polinizadores, un acertado sistema productivo (marcos de plantación y poda 
de formación), un sistema de riego apropiado a las condiciones edafoclimáticas de la zona; entre los más relevantes (Neus, 2011). El agua y los nutrientes constituyen los combustibles más importantes que poseen los cultivos para alcanzar su máximo potencial productivo. Ambos factores no pueden ser considerados como entes independientes entre sí, sino muy al contrario, como factores complementarios de efecto sinérgico. Es a través del agua como los nutrientes se incorporan a la planta para generar, a su vez, un mayor crecimiento vegetativo, productivo y de tamaño del fruto que requerirá una mayor demanda de agua. Sin embargo, el incremento de cosecha no es el único aspecto mejorable. También la menor alternancia y la mejor calidad de los frutos obtenidos serán resultados esperables con un buen manejo del riego y fertilización. En la medida que se alcance el equilibrio entre clima, cultivo, suelo y manejo se conseguirá un mayor o menor acercamiento al máximo potencial productivo (Gispert, 2011). Se debe ejercer un control de plagas muy estricto dado que estas perjudican notablemente la calidad química y el aspecto de los frutos. La poda es un agente de suma importancia en esta etapa de producción, dado que permite a la planta una mejor interceptación y distribución de la luz solar por el follaje; optimizando el rendimiento del proceso de fotosíntesis, el cual deviene en mayor producción y mejor calidad de fruto seco. La recolección en huertos que contienen distintas variedades, se reali- za según las diferentes fechas de maduración con particular consideración para cosechar oportunamente, a fin de evitar el deterioro de los frutos expuestos a la acción de insectos, pájaros y accidentes climáticos, debido a la apertura del capote, característica que depende del cultivar. La cáscara que forma la envoltura exterior es verdosa, más o menos aterciopelada y al llegar la época de madurez, se abre y se deseca. Cada variedad tiene su época completa de maduración. La recogida por vareo tiene el gran inconveniente de que no sólo deja caer al suelo el fruto, sino que a la vez daña ramas que llevarán yemas fructíferas que darán la cosecha el año siguiente. $\mathrm{Si}$ el vareo es enérgico puede ocasionar heridas importantes en las ramas. En lugar del vareo podría retrasarse la recolección un tiempo, pues la nuez y la almendra, mientras están en el árbol, no se perjudican; y cuando están perfectamente maduras caen sacudiendo las ramas con suavidad. Muy pocos productores realizan la cosecha mecánica, dado que para ello hay que contar con un tamaño mínimo de huerto en hectáreas que justifique la inversión de la compra de maquinaria (un tamaño mínimo mayor a $50 \mathrm{ha}$ ), entre otros factores. El primer pelado de las "nuts" consiste en la separación del mesocarpio del endocarpio o cáscara, lo cual se realiza de manera manual o a través de peladoras mecánicas. Los frutos secos, después de su pelado, presentan una humedad aproximada de entre el 12 $25 \%$, por ello conviene secarlas para poder 
conservarlas mejor (humedad inferior al 7 $\%)$. En países cálidos, secos y mediterráneo es suficiente dejarlas secar aire libre durante unos días, aunque para un secado rápido se utilizan secadores industriales constituidos por túneles de aire caliente. Posteriormente, estos frutos se pueden comercializar con $\mathrm{o}$ sin cáscara (endocarpio). Esta segunda etapa de pelado también puede realizarse en forma manual o mecánica.

Asimismo, los residuos originados en estas operaciones pueden ser utilizados como materias primas de otras industrias ya que en el caso de la nuez, por ejemplo, de las dos capas externas del fruto (epicarpio y mesocarpio) se obtiene un colorante denominado "nogalina”, que es muy utilizado para teñir muebles. Las hojas, el epicarpio y mesocarpio contienen abundantes taninos que le confieren su propiedad fuertemente astringente. Además se le puede atribuir propiedades antisépticas, cicatrizantes, tonificantes, vermífugas e hipoglucemiantes (fitoterapia). Otro subproducto que se puede obtener del endocarpio de la nuez es el carbón activado. El proceso general para la obtención de este producto consiste en la carbonización del material, seguido de activación química o física en atmósfera inerte (Martínez et al., 2003, 2006).

La última parte del ciclo productivo primario para la obtención de los frutos secos está dado por su procesamiento, embalaje, posterior almacenamiento y utilización en distintas formas, de acuerdo al uso o destino previsto de las mismas. Las principales formas para la utilización de los frutos secos se encuentran estandarizadas a nivel internacional al igual que sus procesos. Estos frutos secos se pueden comercializar: enteros (nuez y almendra), natural o blanqueada (almendra) y en mitades o partidas (nuez). En el caso de las almendras los usos o aplicaciones típicas son: tentempiés naturales, tostados o saborizados; bañadas o cubiertas con chocolate. En el caso de ambos frutos se comercializan como: confituras; ingredientes y rellenos para productos de confitería; barras de cereales; productos de panadería; postres; helados; aderezo para ensaladas; como "snacks" con el agregado de sal y algún condimento; entre otras aplicaciones culinarias.

Como se mencionó al comienzo del trabajo, la nuez y la almendra son ricas en energía ya que contienen entre un 50 a $70 \%$ en peso de aceite el cual se encuentra altamente insaturado y a su vez son fuente de proteínas, hidratos de carbono, fibras, minerales, vitaminas y otros compuestos hidrosolubles como polifenoles y pigmentos.

La comercialización de los frutos secos como tal requiere del cumplimiento de especificaciones pomológicas estandarizadas por la 
normativa vigente en el mercado nacional e internacional (tamaño, color, presencia de defectos como manchas, color irregular, vestigios de contaminación microbiana, entre otros). En este contexto, aquellas semillas que no cumplen con los parámetros especificados no son aptas para consumo directo y se transforman en material de "descarte" en la cadena de valor tradicional. Sin embargo, estas semillas son aptas para la obtención de productos no tradicionales y altamente apreciados por su calidad nutricional y sensorial, como lo son el aceite y la harina, los cuales le otorgan mayor valor agregado a aquellas semillas.

De acuerdo al volumen de producción y a las cualidades físicas (tamaño y dureza) de las semillas de nuez y almendra, se constituyen en un material apropiado para la extracción del aceite por prensado, una tecnología que no requiere de solventes y que por lo tanto no genera residuos de ese tipo. Un aspecto muy importante a considerar para la utilización de la tecnología de prensado es que el material debe ser acondicionado previamente, para lograr condiciones adecuadas (contenido de humedad y temperatura) que permitan optimizar el proceso, en términos de rendimiento y calidad del producto.

\section{CONCLUSIONES}

La extracción por prensado a escala piloto indicó que el contenido de humedad de los frutos resulta un factor fundamental para lograr la máxima recuperación de aceite para ambas drupas. Los aceites obtenidos mediante los distintos tratamientos no presentaron mayores diferencias en su composición química por lo que, en principio, las metodologías utilizadas resultan asequibles para la extracción de aceites de buena calidad.

La producción de estos aceites constituye una alternativa viable para incrementar el valor agregado de estos cultivos, sobre todo con variedades como Franquette (nuez) y Guara (almendra), poco aceptadas para consumo directo. Por sus características físicas y químicas, los aceites de nuez y almendra se pueden consumir de manera directa sin refinar. No obstante, para su conservación y almacenamiento deben ser protegidos con sustancias que puedan inhibir o retardar su enranciamiento. Entre las sustancias ensayadas con tal fin, el TBHQ, solo o combinado, presentó la mayor eficacia antioxidante, aún con la mitad de la concentración máxima permitida por el Código Alimentario Argentino. Los antioxidantes naturales analizados, si bien no lograron la eficacia del TBHQ, ofrecen una alternativa que debe ser explorada con mayor profundidad, teniendo en cuenta efectos sinérgicos que pueden contribuir a estabilizar el aceite utilizando menores cantidades de antioxidantes sintéticos. 
Las pastas formuladas cumplen con las propiedades reológicas, de textura y calidad características de este tipo de productos, tanto para aquellas formuladas en combinación con harina de trigo (parcialmente sustituida) como para las libre de gluten, lo que permitió obtener un producto final con mayor cantidad y calidad proteica; mayor contenido de fibra alimentaria, ácidos grasos esenciales y compuestos con capacidad antioxidante.

\section{REFERENCIAS BIBLIOGRÁFICAS}

AACC (1995). Approved methods of American Association of Cereal Chemists, EUA.

Ahmad, Z. (2010). The uses and properties of almond oil. Complementary Therapies in Clinical Practice, 16, 10-12.

Alasalvar, C., Karamac, M., Amarowicz, R. \& Shahidi, F. (2006). Antioxidant and antiradical activities in extracts of hazelnut kernel (Corylus avellana L.) and hazelnut green leafy cover. Journal of Agricultural and Food Chemistry, 54, 4826-4832.

AOCS (1998). Official Methods and Recommended Practices of the American Oil Chemists' Society ( $5^{\mathrm{a}}$. Ed.). AOCS Press, Champaign, IL, USA.

Brunnel, C., Pareyt, B., Brijs, K. \& Delcour, J.A. (2010). The impact of the protein network on the pasting and cooking properties of dry pasta products. Food Chemistry, 120, 371-378.

Chen, C.Y., Lapsley, K. \& Blumberg, J. (2006). A nutrition and health perspective on almonds. Journal of the Science of Food and Agriculture, 86, 2245-2250.

Crews, C., Hough, P., Godward, J., Brereton, P., Lees, M., Guiet, S. \& Winkelmann, W. (2005). Study of the main constituents of some authentic walnut oils. Journal of Agricultural and Food Chemistry, 53, 4853-4860.

Dick, J.W. \& Matsuo, R.R. (1988). Durum wheat and pasta products. In: Wheat: Chemistry and Technology. Vol 2. ( $3^{\text {rd }}$ Ed.). American Association of Cereal Chemists, St. Paul MN.

Frankel, E.N. (2005). Lipid oxidation. Barnes, P.J. (Ed.). The Oily Press. Bridgwater, England. 
Gispert, J.R. (2011). Seminario Internacional de Frutos Secos, Choele Choel, Rio Negro, Argentina.

Goli, A. H., Barzegar, M. \& Sahari, M. A. (2005). Antioxidant activity and total phenolic compounds of pistachio (Pistachia vera) hull extracts. Food Chemistry, 92, 521-525.

Gu, L., Kelm, M. A., Hammerstone, J. F., Beecher, G., Holden, J., Haytowitz, D. et al. (2003). Screening of foods containing proanthocyanidins and their structural characterization using LC-MS/MS and thiolytic degradation. Journal of Agricultural and Food Chemistry, 51, 75137521.

Hadad, L., Menapace, E., Maestri, D., Penci, C \& Martínez, M.L. (2012, marzo). Extracción de aceite de almendra mediante prensado: estudio de algunas variables del proceso y su relación con el rendimiento y la calidad del mismo. Congreso Latinoamericano de Ingeniería y Ciencias Aplicadas, San Rafael, Mendoza, Argentina.

Horn, A.F., Nielsen, N.S. \& Jacobsen, C. (2009). Additions of caffeic acid, ascorbyl palmitate or g-tocopherol to fish oil-enriched bars affect lipid oxidation differently. Food Chemistry, 112, 412-420.

Kamath, V. \& Rajini, P. S. (2007). The efficacy of cashew nut (Anacardium occidentale L.) skin extract as a free radical scavenger. Food Chemistry, 103, 428-433.

Kodad, O. \& Socias, I.C.R. (2008). Variability of oil content and of major fatty acid composition in almond (Prunus amygdalus Batsch) and its relationship with kernel quality. Journal of Agricultural and Food Chemistry, 56, 4096-4101.

Labuckas, D.O., Maestri, D.M., Perelló, M., Martínez, M.L. \& Lamarque, A.L. (2008). Phenolics from walnut (Juglans regia L.) kernels: antioxidant activity and interactions with proteins. Food Chemistry, 107, 607-612.

Lavedrine, F., Ravel, A., Villet, A., Ducros, V. \& Alary, J. (2000). Mineral composition of two walnut cultivars originating in France and California. Food Chemistry, 68, 347-351.

Volumen No. 1 Aportes a la sustentabilidad de la cadena de valor de frutos secos: obtención de aceite y harina de nuez (Juglans regia L.) y almendra (Prunus amygdalus L.) 
Lavedrine, F., Zmirou, D., Ravel, A., Balducci, F. \& Alary, J. (1999). Blood cholesterol and walnut consumption: a cross-sectional survey in France. Preventive Medicine, 28, 333-339.

Li, L., Tsao, R., Yang, R., Liu, C., Zhu, H. \& Young, J.C. (2006). Polyphenolic profiles and antioxidant activities of heartnut (Juglans ailanthifolia var. cordiformis) and Persian walnut (Juglans regia L.). Journal of Agricultural and Food Chemistry, 54, 8033-8040.

Li, M., Bellmer, D.D. \& Brusewitz, G.H. (1999). Pecan kernel breakage and oil extracted by supercritical $\mathrm{CO}_{2}$ as affected by moisture content. Journal of Food Science, 64, 1084-1088.

Luis, I. (2011). Seminario Internacional de Frutos Secos, Choele Choel, Rio Negro, Argentina. Maestri, D.M., Nepote, V., Lamarque, A.L. \& Zygadlo, J.A. (2006). Natural products as antioxidants. En: Phytochemistry: Advances in Research. Editor: Filippo Imperato. Research Signpost, Trivandrum, Kerala, India. (pp.105-135).

Martínez, M. L., Moiraghi, L., Agnese, M. \& Guzmán, C. (2003). Making and some properties of activated carbon produced from agricultural industrial residues from Argentina. The Journal of the Argentine Chemical Society, 91(4/6), 103-108.

Martínez, M.L. \& Maestri, D.M. (2008). Oil chemical variation in walnut (Juglans regia L.) genotypes grown in Argentina. European Journal of Lipid Science and Technology, 110, 11831189.

Martínez, M.L., Mattea, M. \& Maestri, D.M. (2006). Varietal and crop year effects on lipid composition of walnut (Juglans regia) genotypes. Journal of the American Oil Chemists' Society, $83,791-796$.

Martínez, M.L., Mattea, M. \& Maestri, D.M. (2008). Pressing and supercritical carbon dioxide extraction of walnut oil. Journal of Food Engineering, 88, 399-404.

Martínez, M.L., Penci, C., Ixtaína, V., Hadad, L., Ribotta, P.D. \& Maestri, D.M. (2012, marzo). Antioxidantes naturales: efecto sobre la estabilidad oxidativa del aceite de nuez (Juglans regia L.). Congreso Latinoamericano de Ingeniería y Ciencias Aplicadas, San Rafael, Mendoza, Argentina. 
Martínez, M.L., Torres, M.M., Guzmán, C.A. \& Maestri, D. M. (2006). Preparation and characteristics of activated carbon from olive stones and walnut shells. Industrial Crops and Products, 23, 23-28.

Menapace, E., Hadad, L., Maestri, D., Martínez, M.L. \& Penci, C. (2012, marzo).Estabilidad y conservación del aceite de almendra obtenido por prensado en frio. Congreso Latinoamericano de Ingeniería y Ciencias Aplicadas, San Rafael, Mendoza, Argentina.

MinAgri (2011), http://www.alimentosargentinos.gov.ar/contenido/sectores/frutasecas / productos/Almendras_2003/Almendras.htm; http://www.alimentosargentinos gov.ar/ contenido/revista/ediciones/45/cadenas/Frutas secas Nuez nogal.html

Moure, A., Domínguez, H., Zúñiga, M.E., Soto, C. \& Chamy, R. (2002). Characterisation of protein concentrates from pressed cakes of Guevina avellana (Chilean hazelnut). Food Chemistry, 78, 179-186.

Moure, A., Franco, D., Sineiro, J., Domínguez, H., Núñez, M. J. \& Lema, J. M. (2000). Evaluation of extracts from Gevuina avellana hulls as antioxidants. Journal of Agricultural and Food Chemistry, 48, 3890-3897.

Neus, A. (2011). Seminario Internacional de Frutos Secos, Choele Choel, Rio Negro, Argentina

Nielsen, N.S. \& Jacobsen, C. (2009). Methods for reducing lipid oxidation in fish-oil-enriched energy bars. International Journal of Food Science \& Technology, 44, 1536-1546.

Ozcan, M.M., Unver, A., Erkan, E. \& Arslan, D. (2011). Characteristics of some almond kernel and oils. Scientia Horticulturae, 127, 330-333.

Petitot, M., Abecassis, J. \& Micard, V. (2009). Structuring of pasta components during processing: impact on starch and protein digestibility and allergenicity. Trends in Food Science y Technology, 20, 521-532.

Prasad, R.B.N.(2003). Walnuts and pecans. En: Encyclopedia of Food Science and Nutrition. Academic Press, London (pp. 6071-6079). 
Singh, J. \& Bargale, P.C. (2000). Development of a small capacity double stage compression screw press for oil expression. Journal of Food Engineering, 43(2), 75-82.

Singh, K.K., Wiesenborn, D.P., Tostenson, K. \& Kangas, N. (2002). Influence of moisture content and cooking on screw pressing of crambe seed. Journal of the American Oil Chemist's Society, 79(2), 165-170.

Singh, K.K., Wiesenborn, D.P., Tostenson, K. \& Kangas, N. (2002). Influence of moisture content and cooking on screw pressing of crambe seed. Journal of the American Oil Chemist's Society, 79(2), 165-170.

Soto, C, Chamy, R.L. \& Zuniga, M.E. (2007). Enzymatic hydrolysis and pressing conditions effect on borage oil extraction by cold pressing. Food Chemistry, 102(3), 834-40.

Sze-Tao, K.W.C. \& Sathe, S.K. (2000). Walnut (Juglans regia L.): Proximate composition, protein solubility, protein amino acid composition and protein in vitro degestability. Journal of the Science of Food and Agriculture, 80, 1393-1401.

Tudorica, C.M., Kuri, V. \& Brennan CS. (2002). Nutritional and Physicochemical Characteristics of Dietary Fiber Enriched Pasta. Journal of Agricultural and Food Chemistry, 50, 347-356.

Uquiche, E., Jeréz, M. \& Ortíz, J. (2008). Effect of pretreatment with microwaves on mechanical extraction yield and quality of vegetable oil from Chilean hazelnuts (Gevuina avellana Mol). Innovative Food Science and Emerging Technologies, 9, 495-500.

Vargas-Lopez, J.M., Wiesenborn, D., Tostenson, K. \& Cihacek, L. (1999). Processing of crambe for oil and isolation of erucic acid. Journal of the American Oil Chemist's Society, 76, 801-809.

Verardo, V., Ferioli, F., Riciputi, Y., Iafelice, G., Marconi, E. \& Caboni, M.F. (2009). Evaluation of lipid oxidation in spaghetti pasta enriched with long chain n-3 polyunsaturated fatty acids under different storage conditions. Food Chemistry, 114, 472-477.

Wardlaw, G.M. (1999). Perspectives in nutrition. McGraw-Hill, New York, USA.

Marcela Lilian Martínez / María Cecilia Penci / Damián Modesto Maestri / Pablo Daniel Ribotta / María Andrea Marín | Volumen No. 1 
Wiesenborn, D., Doddapaneni, R., Tostenson, K. \& Kangas, N. (2001). Cooking indices to predict screw-press performance for crambe seed. Journal of the American Oil Chemist's Society, 78(5), 467-471.

Wong, D.W.S. (1995). Lípidos. En: Química de los alimentos: Mecanismos y teoría. Ed. Acribia, Zaragoza, España (pp. 1-52).

Yanishlieva, N.V. \& Marinova, E.M. (2001). Stabilization of edible oils with natural antioxidants. European Journal of Lipid Science and Technology, 103, 752-767.

Yu, J., Ahmedna, M. \& Goktepe, I. (2005). Effects of processing methods and extraction solvents on concentration and antioxidant activity of peanut skin phenolics. Food Chemistry, 90, 199206.

Zhang, Z., Liao, L., Moore, J., Wu, T. \& Wang, Z. (2009). Antioxidant phenolic compounds from walnut kernels (Juglans regia L.). Food Chemistry, 113, 160-165.

Zheng, Y., Wiesenborn, D.P., Tostenson, K. \& Kangas, N. (2003). Screw pressing of whole and dehulled flaxseed for organic oil. Journal of the American Oil Chemist's Society, 80(10), 10391045.

Zúñiga, M.E., Soto, C., Mora, A., Chamy, R., Lema J.M..(2003). Enzymic pre-treatment of Guevina avellana mol oil extraction by pressing. Process Biochemistry, 39, 51-57.

Volumen No. 1 Aportes a la sustentabilidad de la cadena de valor de frutos secos: obtención de aceite y harina de nuez (Juglans regia L.) y almendra (Prunus amygdalus L.) 